\title{
A Stabilizing Agent, PCA/DTPA, Improves Plasma Storage Life for the Chromsystems Vitamin C Assay up to Six Months
}

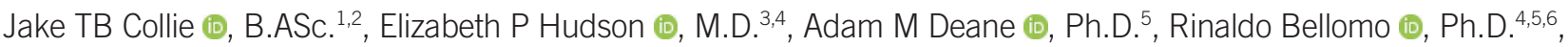 \\ and Ronda F Greaves (D), Ph.D. 1,2,7,8 \\ ${ }^{1}$ School of Health and Biomedical Sciences, RMIT University, Bundoora, Victoria, Australia; ${ }^{2}$ Royal College of Pathologists Quality Assurance Programs \\ Vitamins Advisory Committee, Sydney, New South Wales, Australia; ${ }^{3}$ Dubbo Hospital, Western NSW Local Health District, Dubbo, New South Wales, \\ Australia; ${ }^{4}$ Department of Critical Care, The University of Melbourne, Melbourne, Australia; ${ }^{5}$ Department of Intensive Care, Royal Melbourne Hospital, \\ Parkville, Victoria, Australia; ${ }^{6}$ Department of Intensive Care, Austin Health, Heidelberg, Australia; ${ }^{7}$ Victorian Clinical Genetics Services, Murdoch Children's \\ Research Institute, Parkville, Victoria, Australia; ${ }^{8}$ Department of Paediatrics, University of Melbourne, Melbourne, VIC, Australia
}

The commonly used Chromsystems vitamin C (ascorbate) assay (Munich, Germany) has a sample storage life of five days at $-20^{\circ} \mathrm{C}$. Stabilizing agents have been successfully used to increase longevity; however, their suitability with this commercial assay is unclear. We investigated the compatibility of a stabilizing agent, perchloric acid/diethylenetriaminepentaacetic acid (PCA/DTPA), with the Chromsystems assay. Plasma was stored at $-80^{\circ} \mathrm{C}$, with or without PCA/DTPA. Storage up to six months was assessed through baseline and repeat analyses, stability was assessed by comparing paired non-stabilized and PCA/ DTPA-stabilized plasma, and performance was assessed using allowable performance specifications of an external quality assurance program. Ascorbate concentration was significantly lower in non-stabilized plasma than in paired PCA/DTPA-stabilized plasma, with a proportional difference of $11 \%(P=0.01)$. All storage analysis results were within the allowable performance specifications. Storage at $-80^{\circ} \mathrm{C}$ prevented plasma ascorbate oxidation; however, substantial oxidation occurred during sample processing. In conclusion, PCA/DTPA significantly reduces ascorbate oxidation, and PCA/DTPA-stabilized ascorbate plasma is compatible with the Chromsystems assay and stable for up to six months, when stored at $-80^{\circ} \mathrm{C}$.

Key Words: Ascorbate, Vitamin C, Storage, Stability, Chromsystems, PCA/DTPA
Received: July 7, 2020

Revision received: September 14, 2020

Accepted: January 12, 2021

\section{Corresponding author:}

Ronda Greaves, Ph.D.

The Murdoch Children's Research Institute, 50 Flemington Rd, Parkville VIC 3052,

Australia

Tel: +61-03-8341-6272

Fax: +61-03-8341-6339

E-mail: ronda.greaves@mcri.edu.au

\section{(c) (i) (5)}

(c) Korean Society for Laboratory Medicine This is an Open Access article distributed under the terms of the Creative Commons Attribution Non-Commercial License (https://creativecommons.org/licenses/by-nc/4.0) which permits unrestricted non-commercial use, distribution, and reproduction in any medium, provided the original work is properly cited.
There has been a resurgence of studies on vitamin C (ascorbate), particularly in relation to critical care, which has led to an increased interest in its measurement [1]. According to the external proficiency testing program run by the Royal College of Pathologists of Australasia Quality Assurance Programs (RCPAQAP), a commercial assay supplied by Chromsystems (Munich, Germany) is the most widely used method to measure ascorbate in serum or plasma [2]. The assay combines high-performance liquid chromatography (HPLC) with ultra-violet (UV) detection. The assay instructions state that the durability of plasma ascor- bate is five days when stored at $-20^{\circ} \mathrm{C}$. This is prohibitively short for studies using plasma that is collected over a long period and batch-analyzed for randomized control trials (RCTs) and plasma collected in remote areas.

Methods to improve the ascorbate stability by minimizing its oxidation to dehydroascorbic acid (DHA) using a stabilizing agent or by reducing DHA to ascorbate have been published previously [3-8]. These studies used in-house analytical methods to validate the sample handling and processing procedures. However, there is no evidence to support the application of the sam- 
ple processing procedures to the popular commercial assay. Therefore, we determined (1) whether the inclusion of a stabilizing agent during sample handling and processing is compatible with the commercial assay and, if it is, (2) whether plasma ascorbate stability can be extended beyond the period recommended by the manufacturer.

This study was nested within the Vitamin C, Hydrocortisone and Thiamine in Patients With Septic Shock (VITAMINS) trial, NCT03333278 [9] (ethics approval number: HREC/17/Austin/238). The RCT was conducted over 18 months from May 2018. Informed consent was obtained from the participants. Blood samples were collected in lithium heparin vacutainer tubes (BD, Franklin Lakes, NJ, USA, cat. no. 367886) and centrifuged at $4,000 \mathrm{rpm}(1,431 \times \mathrm{g})$ and $4^{\circ} \mathrm{C}$ for 10 minutes. Plasma from each sample was transferred into multiple $1.7 \mathrm{~mL}$ microfuge tubes (cat. number AX-MCT-175-C; Fisher BioTeC, Wembley, WA, Australia) and subjected to two sample handling and processing methods. One group of plasma samples was immediately stored at $-80^{\circ} \mathrm{C}$ (Fig. $1 \mathrm{~A}$ ), and, in the other group, a stabilizing agent, perchloric acid (PCA, cat. number D6518; SigmaAldrich, Castle Hill, NSW, Australia)/diethylenetriaminepentaacetic acid (DTPA, cat. number 311421; Sigma-Aldrich), was added at a plasma:PCA/DTPA ratio of 1:1 (v:v) prior to storage at $-80^{\circ} \mathrm{C}$ (Fig. 1B). The study design considered the expected available plasma volume from the critical care patient popula- tion, which led to the decision to assess one stabilizing reagent, PCA/DTPA.

PCA/DTPA was selected owing to its success in minimizing ascorbate oxidation to DHA [3]. PCA/DTPA was prepared at a concentration of $0.54 \mathrm{M}$ by adding $4.66 \mathrm{~mL}$ of $70 \%$ PCA to 100 $\mathrm{mL}$ of deionized water, followed by the addition of $10 \mathrm{mg}$ of DTPA, and was stored at $4^{\circ} \mathrm{C}$. The above-cited study recommended plasma storage at $-80^{\circ} \mathrm{C}$. We did not assess the storage temperature of $-20^{\circ} \mathrm{C}$ as advised in the commercial assay instructions because some studies have reported that non-stabilized plasma stored at temperatures above $-75^{\circ} \mathrm{C}$ exhibits a higher oxidation rate $[8,10]$. Additionally, in studies using an acid stabilizer and metal chelator, such as PCA/DTPA, plasma was stored at $-80^{\circ} \mathrm{C}[5,11]$.

Plasma samples were analyzed on a Shimadzu HPLC-UV system (Rydalmere, NSW, Australia). The Chromsystems assay, reagents, column, and internal quality controls (IQCS) were sourced by Astral Scientific (Taren Point, NSW, Australia). Performance was assessed by the inclusion of a bi-level IQC run within each batch, as well as enrollment into the RCPAQAP plasma vitamin $\mathrm{C}$ program. The average coefficient of variation $(\mathrm{CV})$ of the bilevel IQC (mean low concentration=37.1; high concentration= $111.9 \mu \mathrm{mol} / \mathrm{L}$ ) throughout this study was $9.6 \%$ ( $\min =8.1 \%$; $\max =11.0 \%$; uncertainty of measurement $=22.0 \%$ ), while RCPAQAP yielded a CV of $3.9 \%$ (based on the median results of

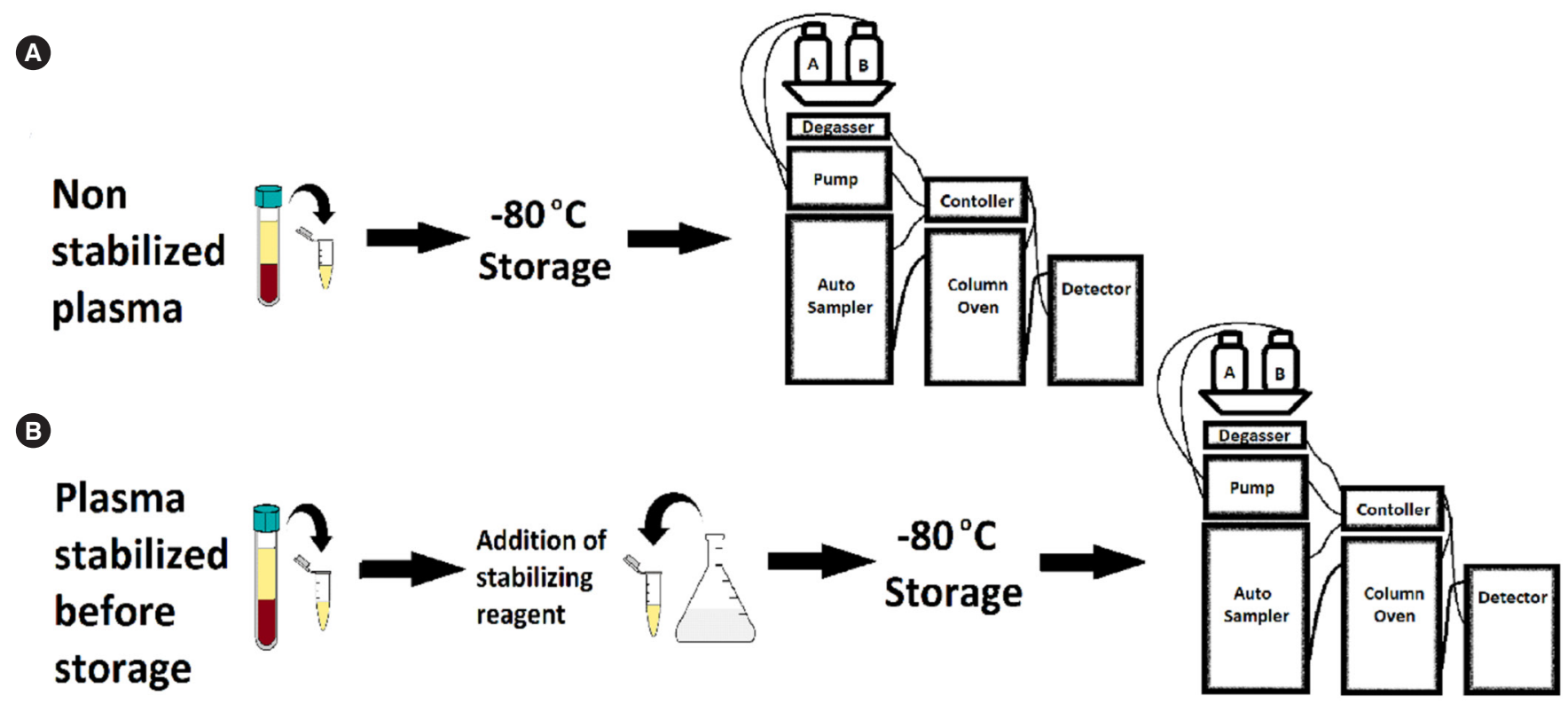

Fig. 1. Schematic representation of the plasma sample handling and processing procedures utilized in this study. (A) Immediate storage of non-stabilized plasma at $-80^{\circ} \mathrm{C}$, followed by HPLC-UV analysis. (B) Addition of the PCA/DTPA stabilizing agent to the plasma aliquot (1:1, $\mathrm{v}: \mathrm{V}$ ) and storage at $-80^{\circ} \mathrm{C}$, followed by HPLC-UV analysis.

Abbreviations: PCA, perchloric acid; DTPA, diethylenetriaminepentaacetic acid; HPLC-UV, high performance liquid chromatography with ultra-violet detection. 
22 participants; $\min =2.4 ; \max =17.5 \%$ ) and a bias of $5.0 \mu \mathrm{mol} / \mathrm{L}$ ( $\min =0.7 ; \max =18.8 \mu \mathrm{mol} / \mathrm{L})$. The allowable performance specifications (APS) set out by the RCPAQAP are based on biological variation studies and were adopted in this study to determine significant differences [2, 12].

Three experiments were performed to assess the storage and stability of ascorbate whilst validating the PCA/DTPA compatibility with the Chromsystems assay. The first experiment was a storage study of non-stabilized plasma samples $(\mathrm{N}=39)$, wherein we measured the baseline ( $\left.T_{0}\right)$ ascorbate concentration and repeated the analysis of the duplicate samples $\left(T_{1}\right) 2,7,15$, or 26 weeks ( \pm 1 week) later. The results of $T_{1}$ and $T_{0}$ were compared to assess significant changes in concentration. The second experiment was identical to the first one, except that PCA/DTPAstabilized plasma samples $(\mathrm{N}=42)$ were analyzed. In the third

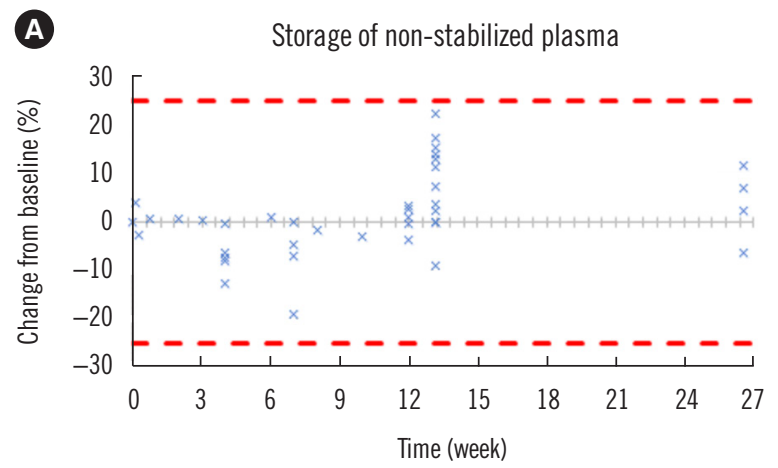

○

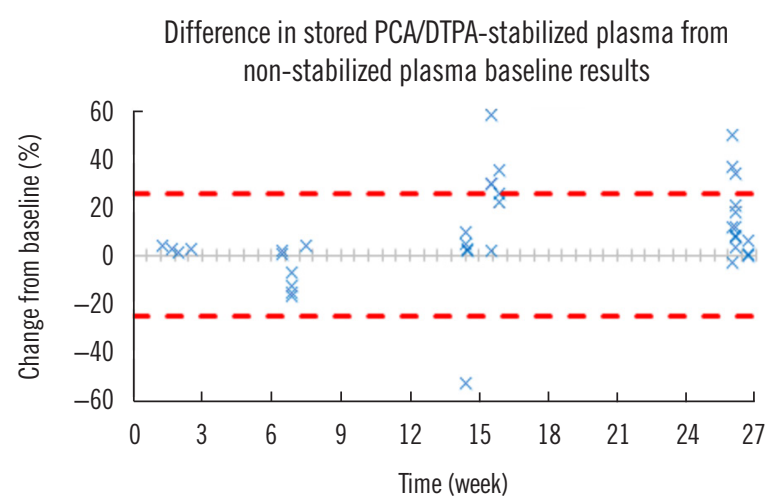

experiment, we compared non-stabilized and paired PCA/DTPAstabilized plasma samples. Time points of 2, 7, 15, and 26 weeks ( \pm 1 week) of storage were selected for analysis to represent expected potential delays associated with the factor combination of recruitment, efficient use of consumables, and instrument availability. Prior to the experiments, PCA/DTPA was diluted with de-ionized water $(1: 1, v: v)$ and analyzed as a blank sample to ensure there was no chromatographic interference.

Statistical tests were carried out and all plots were generated using Microsoft Excel (Washington, DC, USA), with the Analyseit statistical software (Leeds, UK) and MedLabQC software (Metz, France). Paired two-tailed $t$-test, Passing-Bablok regression, and difference plot were used, with To being the independent variable for the storage experiments and non-stabilized plasma for the comparison experiment. Results were considered statis-
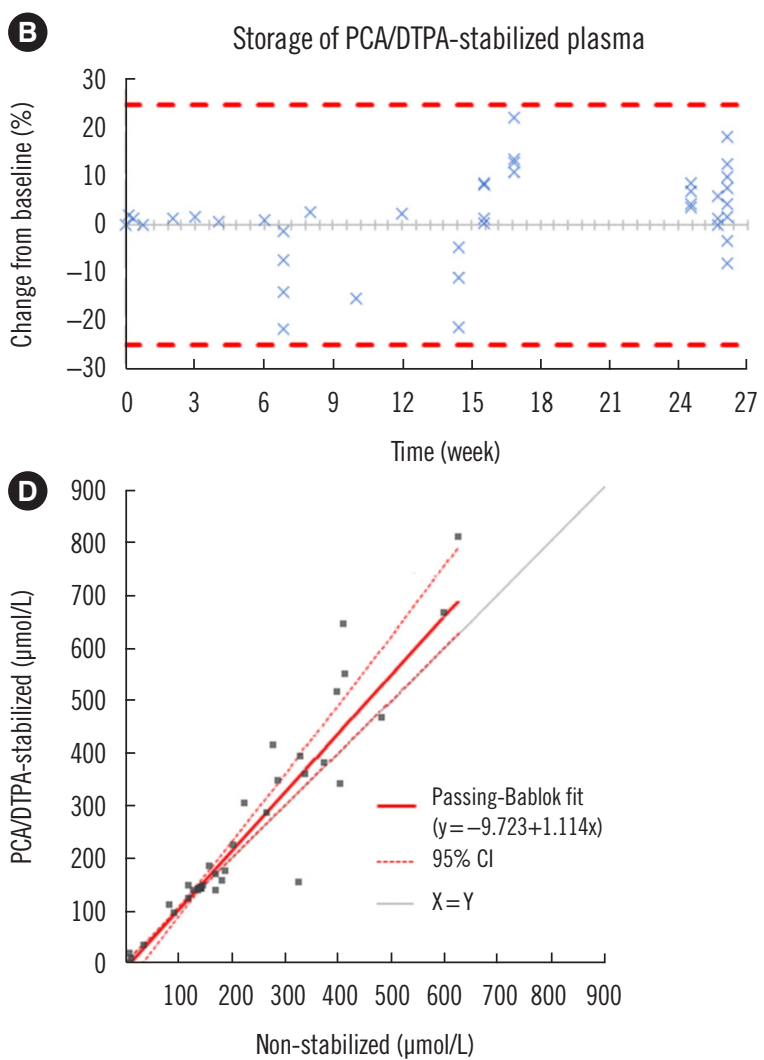

Fig. 2. Difference plots and Passing-Bablok regression of storage and stability experiments. The data displayed in panels $A$, B, and $C$ are results from the $T_{1}$ analyses for each plasma sample and are expressed as a percentage change in concentration from the baseline, $T_{0}$, on the $Y$-axis. The $X$-axis represents weeks in storage before the $T_{1}$ analysis. (A) Storage of non-stabilized plasma. Mean percentage concentration difference of $+1.2 \%$ (median+0.5\%). (B) Storage of PCA/DTPA-stabilized plasma. Mean percentage concentration difference of $-0.2 \%$ (median+1.5\%). (C) Comparison of PCA/DTPA-stabilized plasma with paired non-stabilized plasma. Numbers of samples per time point analyzed: 2 weeks $(N=4), 7$ weeks $(N=7), 15$ weeks $(N=14)$, and 26 weeks $(N=14)$. Mean percentage concentration difference of $+9.9 \%$ (median+3.6\%). (D) Passing-Bablok regression of non-stabilized plasma against PCA/DTPA-stabilized plasma. Concentrations determined through all experiments ranged from $2-812 \mu \mathrm{mol} / \mathrm{L}$. The dashed lines in panels $\mathrm{A}$, B, and $\mathrm{C}$ represent the $\pm 25 \%$ APS for results $\geq 36.0 \mu \mathrm{mol} / \mathrm{L}$.

Abbreviations: PCA, perchloric acid; DTPA, diethylenetriaminepentaacetic acid; APS, allowable performance specifications; Cl, confidence interval. 
tically significant when $P \leq 0.05$, the $95 \%$ confidence interval (Cl) of the difference plot did not pass through zero, or the 95\% $\mathrm{Cl}$ of the Passing-Bablok slope did not include 1.00.

The two storage experiments revealed no significant change in ascorbate concentration between non-stabilized $(P=0.98)$ and PCA/DTPA-stabilized $(P=0.15)$ plasma over six months when using the Chromsystems assay. A non-significant bias was observed in the difference plots for these experiments, with an estimated difference of $-0.1 \mu \mathrm{mol} / \mathrm{L}(95 \% \mathrm{Cl}=-9.6-9.3 \mu \mathrm{mol} / \mathrm{L})$ for non-stabilized plasma and $5.7 \mu \mathrm{mol} / \mathrm{L}(95 \% \mathrm{Cl}=-2.1-13.5$ $\mu \mathrm{mol} / \mathrm{L}$ ) for PCA/DTPA-stabilized plasma. All results were within the APS (Fig. 2A and B).

The comparison experiment revealed that the PCA/DTPA-stabilized plasma had a significantly higher ascorbate concentration $(P=0.01)$ than the non-stabilized plasma with an average difference of $27 \mu \mathrm{mol} / \mathrm{L}$ ( $\mathrm{Min}=-6$, Max $=235 \mu \mathrm{mol} / \mathrm{L}$ ) when using the Chromsystems assay. Multiple data points fell outside the APS limits, and the average percentage difference between the two groups was 10\% (Fig. 2C). The Passing-Bablok regression analysis revealed a significant slope of $1.11(95 \% \mathrm{Cl}=1.02$ 1.29), indicating a $11 \%$ proportional difference between the paired PCA/DTPA-stabilized and non-stabilized plasma, and a non-significant intercept of $-9.72(95 \% \mathrm{Cl}=-34.93-2.57 \mu \mathrm{mol} / \mathrm{L})$ (Fig. 2D).

Our results indicate that plasma ascorbate concentration stabilized with PCA/DTPA is compatible with the Chromsystems assay. In addition, plasma stored at $-80^{\circ} \mathrm{C}$ was stable for up to six months, regardless of the addition of PCA/DTPA. This is considerably longer than the stability of five days at $-20^{\circ} \mathrm{C}$ reported by the manufacturer. Prolonged storage life will be suitable for time-sensitive analyses conducted over long durations and remotely collected samples that require transportation and will allow batch analyses in routine pathology laboratories and RCTs.

PCA/DTPA prevents the oxidation and, ultimately, hydrolysis, of ascorbate. A significant loss of ascorbate would be expected in the absence of a stabilizer at room temperature. A significant loss of ascorbate has been reported in samples incubated at $23-25^{\circ} \mathrm{C}$ for 2 hours, and a median reduction in ascorbate concentration of $18 \%$ (range $=10 \%-32 \%$ ) was observed after a 2-hour delay of plasma separation [5, 13]. However, when a stabilizing agent is added, the amount of ascorbate oxidized to DHA is minimized [5].

The two storage experiments demonstrated that plasma was stable for up to six months when stored at $-80^{\circ} \mathrm{C}$. This suggests that storage at ultra-low temperature does not cause a significant loss of analyte through oxidation. In the storage experiment us- ing PCA/DTPA-stabilized plasma, owing to the suppression of ascorbate oxidation, the analyte concentration was maintained throughout the study period, which led to a non-significant change in results. In non-stabilized plasma, ascorbate oxidation occurred until the plasma was prepared for $T_{0}$ analysis or reached a frozen state, resulting in an underestimation of the actual ascorbate concentrations.

As the Chromsystems assay measures only ascorbate concentration, not DHA, the differences observed in the comparison experiment are likely due to the minimization of ascorbate oxidation by PCA/DTPA. Therefore, a higher ascorbate concentration was maintained in the PCA/DTPA-stabilized plasma than in the paired non-stabilized plasma. Although the Passing-Bablok regression analysis revealed a significant slope, the percentage was within the uncertainty of measurement of $22 \%$ and unlikely to be clinically significant. Therefore, we predict that the use of the PCA/DTPA would not affect the reference values and decision limits, provided that sample handling and processing procedures remain consistent.

Our experiments provided evidence that the inclusion of the PCA/DTPA stabilizing agent reduces analyte loss by minimizing ascorbate oxidation during sample handling and processing, which are conducted at temperatures higher than the storage temperature.

Plasma ascorbate stability has also been demonstrated in a previous study, using other reagents and sample handling and processing procedures [1]. However, these procedures had not been verified for the Chromsystems assay. Hence, our work validates the compatibility of PCA/DTPA for sample handling and processing with this assay. This information is valuable for laboratories employing the Chromsystems assay as well as for RCTs on ascorbic acid intervention using plasma ascorbate analysis.

We used samples collected from patients in an intensive care setting since it reflects real patient results and demonstrates clinical utility in the target population. However, because of the small study population, we could assess only one stabilizing agent and one storage temperature. Despite the perceived limitations, the results obtained sufficiently provided evidence for the compatibility of PCA/DTPA with the Chromsystems assay and demonstrated a significant difference in ascorbate concentrations between non-stabilized and PCA/DTPA-stabilized plasma with stability longer than the five days recommended by the manufacturer. Our results warrant a repeat study with multiple time points and other stabilizing or reducing agents and storage temperatures. In addition, a long-term trial to assess analyte stability for a minimum of 1-2 years would be beneficial for creating a 
sample biobank for future clinical studies.

In conclusion, we demonstrated that $-80^{\circ} \mathrm{C}$ storage prevents plasma ascorbate oxidation and that substantial oxidation occurs during sample handling and processing. We verified that the inclusion of PCA/DTPA as a stabilizing agent significantly reduces ascorbate oxidation, allowing for more accurate results, while maintaining compatibility with the Chromsystems assay. We also demonstrated that plasma has a storage life of up to six months at $-80^{\circ} \mathrm{C}$, which is beyond the manufacturer's recommendation. We recommend the inclusion of PCA/DTPA as a stabilizing agent and storage at $-80^{\circ} \mathrm{C}$ for plasma to be analyzed using the Chromsystems assay.

\section{ACKNOWLEDGEMENTS}

We thank the ICU staff at Austin Health and the Royal Melbourne Hospital for their hard work regarding the ethics approval, patient recruitment, sample collection, and processing.

\section{AUTHOR CONTRIBUTIONS}

Collie JTB: Conceptualization, data curation, formal analysis, methodology, investigation, resources, writing original draft as well as editing and reviewing. Hudson EP: Data curation, investigation, resources, editing and reviewing. Deane AM: Conceptualization, investigation, resources, editing and reviewing. BelIomo R: Conceptualization, funding acquisition, investigation, resources, editing and reviewing. Greaves RF: Conceptualization, investigation, project administration, resources, supervision, writing original draft as well as editing and reviewing.

\section{CONFLICTS OF INTEREST}

None declared.

\section{RESEARCH FUNDING}

This project was funded through the Austin Health Intensive Care Trust Fund.

\section{ORCID}

Jake T B Collie

https://orcid.org/0000-0002-4722-6395

Elizabeth P Hudson https://orcid.org/0000-0001-7134-1556

Adam M Deane https://orcid.org/0000-0002-7620-5577

Rinaldo Bellomo https://orcid.org/0000-0002-1650-8939

Ronda F Greaves https://orcid.org/0000-0001-7823-8797

\section{REFERENCES}

1. Collie JTB, Greaves RF, Jones OAH, Eastwood GM, Bellomo R. Vitamin $\mathrm{C}$ measurement in critical illness: challenges, methodologies and quality improvements. Clin Chem Lab Med 2020;58:460-70.

2. RCPA Quality Assurance Programs. Vitamin C End of Cycle 41 Report. https://myqap.rcpaqap.com.au/reports (Updated on Dec 2019).

3. Salminen I and Alfthan G. Plasma ascorbic acid preparation and storage for epidemiological studies using TCA precipitation. Clin Biochem 2008;41:723-7.

4. Karlsen A, Blomhoff R, Gundersen TE. Stability of whole blood and plasma ascorbic acid. Eur J Clin Nutr 2007;61:1233-6.

5. Pullar JM, Bayer S, Carr AC. Appropriate handling, processing and analysis of blood samples is essential to avoid oxidation of vitamin $C$ to dehydroascorbic acid in clinical samples. Antioxidants 2018;7:29.

6. Lykkesfeldt J. Ascorbate and dehydroascorbic acid as reliable biomarkers of oxidative stress: analytical reproducibility and long-term stability of plasma samples subjected to acidic deproteinization. Cancer Epidemiol Biomarkers Prev 2007;16:2513-6.

7. Rossi B, Tittone F, Palleschi S. Setup and validation of a convenient sampling procedure to promptly and effectively stabilize vitamin $C$ in blood and plasma specimens stored at routine temperatures. Anal Bioanal Chem 2016;408:4723-31.

8. Jenab M, Bingham S, Ferrari P, Friesen MD, Al-Delaimy WK, Luben R, et al. Long-term cryoconservation and stability of vitamin $\mathrm{C}$ in serum samples of the European prospective investigation into cancer and nutrition. Cancer Epidemiol Biomarkers Prev 2005;14:1837-40.

9. Fujii T, Udy AA, Deane AM, Luethi N, Bailey M, Eastwood GM, et al. Vitamin C, Hydrocortisone and Thiamine in Patients with Septic Shock (VITAMINS) trial: study protocol and statistical analysis plan. Crit Care Resusc 2019;21:119-25.

10. Bobrowicz E, Naskalski JW, Siedlecki A. Preanalytical factors in human plasma ascorbate assay. Clin Chim Acta 2001;314:237-9.

11. Lykkesfeldt J. Ascorbate and dehydroascorbic acid as biomarkers of oxidative stress: validity of clinical data depends on Vacutainer system used. Nutr Res 2012;32:66-9.

12. Talwar DK, Azharuddin MK, Williamson C, Teoh YP, McMillan DC, St J O'Reilly D. Biological variation of vitamins in blood of healthy individuals. Clin Chem 2005;51:2145-50

13. Ching SYL, Prins AW, Beilby JP. Stability of ascorbic acid in serum and plasma prior to analysis. Ann Clin Biochem 2002;39:518-20. 